\title{
Laboratory-based reflectometer using line spectra of an RF-induced gas-discharge lamp in 30- to 200-nm wavelength range
}

\author{
Yue Yu, ${ }^{a}$ Zeyi Ye, ${ }^{\text {a }}$ i Jiang, ${ }^{a}$ Qianxia Yao, ${ }^{a}$ Shuangying $L i,{ }^{a}$ \\ Jiali Wu, ${ }^{a}$ Jinlong Zhang, ${ }^{a}$ Qiushi Huang, ${ }^{a}$ Chun Xie, ${ }^{\text {b }}$ \\ Andrey Sokolov, ${ }^{\mathrm{c}}$ Hongjun Zhou, ${ }^{\mathrm{d}}$ Tonglin Huo, ${ }^{\mathrm{d}}$ Wenbin Li $\odot,{ }^{\mathrm{a}, *}$ and \\ Zhanshan Wang ${ }^{\text {a,* }}$ \\ ${ }^{a}$ Tongji University, Institute of Precision Optical Engineering, School of Physics Science \\ and Engineering, Key Laboratory of Advanced Micro-Structured Materials MOE, \\ Shanghai, China \\ ${ }^{\mathrm{b}}$ Tongji University, School of Mechanical Engineering, Shanghai, China \\ ${ }^{c}$ Helmholtz-Zentrum Berlin für Materialien und Energie, BESSY-II, Berlin, Germany \\ ${ }^{\mathrm{d} U n i v e r s i t y ~ o f ~ S c i e n c e ~ a n d ~ T e c h n o l o g y ~ o f ~ C h i n a, ~ H e f e i, ~ C h i n a ~}$
}

\begin{abstract}
A laboratory-based reflectometer designed for characterizing the reflectivity of optical coatings in 30- to 200-nm wavelength range was recently developed at IPOE. An RF-produced gas-discharge light source is applied to generate characteristic lines. The light source is mounted on a grazing incident monochromator with a 146-deg deviation angle between the incident and diffracted arms. By precisely adjusting the toroidal grating inside the monochromator chamber, monochromatic lights are acquired through the exit slit. A collimator mirror and two sets of collimation slits with $2 \mathrm{~mm} \times 2 \mathrm{~mm}$ dimension are utilized for reducing the divergence of the beam incident on the sample. A high-precision six-axis translation stage, which allows a heavy sample with a maximum diameter of $100 \mathrm{~mm}$, is used to control positions of the samples and the detector. A chopper disk used both for incident light intensity monitor and signal modulation is placed with an incidence angle of $70 \mathrm{deg}$ relative to the incident light beam. The configuration, adjustment process, and test results of the reflectometer are presented in detail. The experimental reflectivity results for $\mathrm{Al} / \mathrm{LiF} / \mathrm{MgF}_{2}$ film obtained from our laboratory and BESSY-II Synchrotron as well as Hefei Synchrotron Light Source are given and compared for demonstrating the reliability of the system. ( $)$ The Authors. Published by SPIE under a Creative Commons Attribution 4.0 International License. Distribution or reproduction of this work in whole or in part requires full attribution of the original publication, including its DOI. [DOI: 10.1117/1.JATIS .8.1.017002]
\end{abstract}

Keywords: laboratory-based reflectometer; gas-discharge lamp; extreme ultraviolet and far ultraviolet; $\mathrm{Sc} / \mathrm{Si}$ multilayer; $\mathrm{Al} / \mathrm{LiF} / \mathrm{MgF}_{2}$ film.

Paper 21138G received Nov. 2, 2021; accepted for publication Jan. 14, 2022; published online Feb. 10, 2022.

\section{Introduction}

Optical coatings working in extreme ultraviolet (EUV) and far ultraviolet (FUV) wavelength range are applied widely for applications such as material analysis, ${ }^{1,2}$ plasma diagnostics,,${ }^{3,4}$ microscopic imaging, ${ }^{5,6}$ EUV lithography, ${ }^{7,8}$ and astrophysics. ${ }^{9-11}$ Among all the applications, astrophysics is of the particular interest of this research. Due to the fact that core optical elements carried on the satellites require reflective films with good performance, ${ }^{12-14}$ precision characterization of the dedicatedly designed films becomes extremely crucial to the capability of the optics on the EUV and FUV missions. As one of the fundamental properties, reflectivity of the films has aroused considerable attention and need meticulous measurements by reflectometers.

*Address all correspondence to Wenbin Li, wbli@tongji.edu.cn; Zhanshan Wang, wangzs@tongji.edu.cn 
Different from x-ray and UV-infrared wavelength ranges for which integrated and standardized commercial reflectometers have already acquired mature technology supports, ${ }^{15,16}$ in the EUV and FUV band, most commercial reflectometers cannot satisfactorily meet the specific user requirements, especially in the wavelength range below $105 \mathrm{~nm} \cdot{ }^{17,18}$

Reflectometers installed on synchrotron radiation stations provide the most reliable metrology for reflectivity measurements in EUV and FUV band, which are distinguished by their spectral continuity, high-intensity and polarized probe beam as well as excellent angular and spectral resolution. ${ }^{19-26}$ However, tests at synchrotron facilities require significant advanced and cumbersome appointment procedures, and the users are allocated only limited beam-time. Such a long turn-around time makes it impossible to test the innovation coatings in-time and is unacceptable for some films having temporal stability issues, such as $\mathrm{Al} / \mathrm{LiF}$ bilayers. On the other hand, due to the existence of higher diffraction order, reliable approaches are required to suppress higher order effects, such as using absorption filters ${ }^{25,27}$ or extra mirrors in the beam path. ${ }^{20,26,28}$

To meet the research needs, laboratory-based reflectometers have been implemented around the world. ${ }^{29-31}$ The earliest developments of laboratory EUV reflectometers can be traced back to the 1990s, when Nakayama et al., ${ }^{32}$ Gullikson et al., ${ }^{33}$ and Windt et al. ${ }^{34}$ developed reflectometers using laser-produced plasma (LPP) light source. Later on, more and more reflectometers based on LPP light source have been set up in laboratories. However, the usage of LPP light source has some limitations, e.g., the working wavelength range was limited in soft x-ray and EUV bands, and the slight instability from shot to shot would definitely influence the measurement accuracy. Aznárez et al. ${ }^{35}$ set up a reflectometer using a gas-discharged light source, which extended the working wavelength range from 50 to $200 \mathrm{~nm}$ to test FUV multilayers. But the intensity of the probe beam was relatively weak because of technical limitations.

For measuring the reflectivity of films working in EUV and FUV wavelength range, we have implemented a reflectometer utilizing an RF-induced gas-discharge plasma light source, which enjoys the advantages such as high energy coupling efficiency, long working hours, and compatibility with a wide range of experimental conditions, compared with the traditional gas discharge light sources. By changing or mixing the discharging gases, a series of dense characteristic lines covering bands from 30 to $200 \mathrm{~nm}$ could be acquired. Presently, only some relative intense characteristic lines of individually discharged gases are applied to perform angular-dependent reflectivity measurements and there are no higher diffraction orders found to overlap with the applied characteristic lines so far. Therefore, the effects of higher orders can be nearly ignored. Thanks to the technique improvement, the light intensity is much stronger and the emitted plasma is limited in a relatively small solid angle. The discharging process generates temporal quasi-continuous light thus the stability of probe beam is much better compared with LPP light source.

In this paper, the reflectometer system is described in detail including the system configuration, the adjusting methods, the measurement procedures as well as the comparison of our measurement results with the data obtained at BESSY-II Synchrotron and Hefei Synchrotron Light Source (HLS) for different samples.

\section{Description of the Reflectometer}

\subsection{Scheme of the System}

The system consists of several basic units as follows: (1) an RF-produced gas-discharge light source, (2) a monochromator with two switchable varied-line-spacing toroidal gratings of 250 and $950 \mathrm{gr} / \mathrm{mm}$, respectively, (3) a collimator chamber, (4) a modulation chamber, and (5) a sample chamber, as showed in Fig. 1. In the following, the structure and the functions of each unit will be explained in detail. 
Yu et al.: Laboratory-based reflectometer using line spectra of an RF-induced gas-discharge lamp...

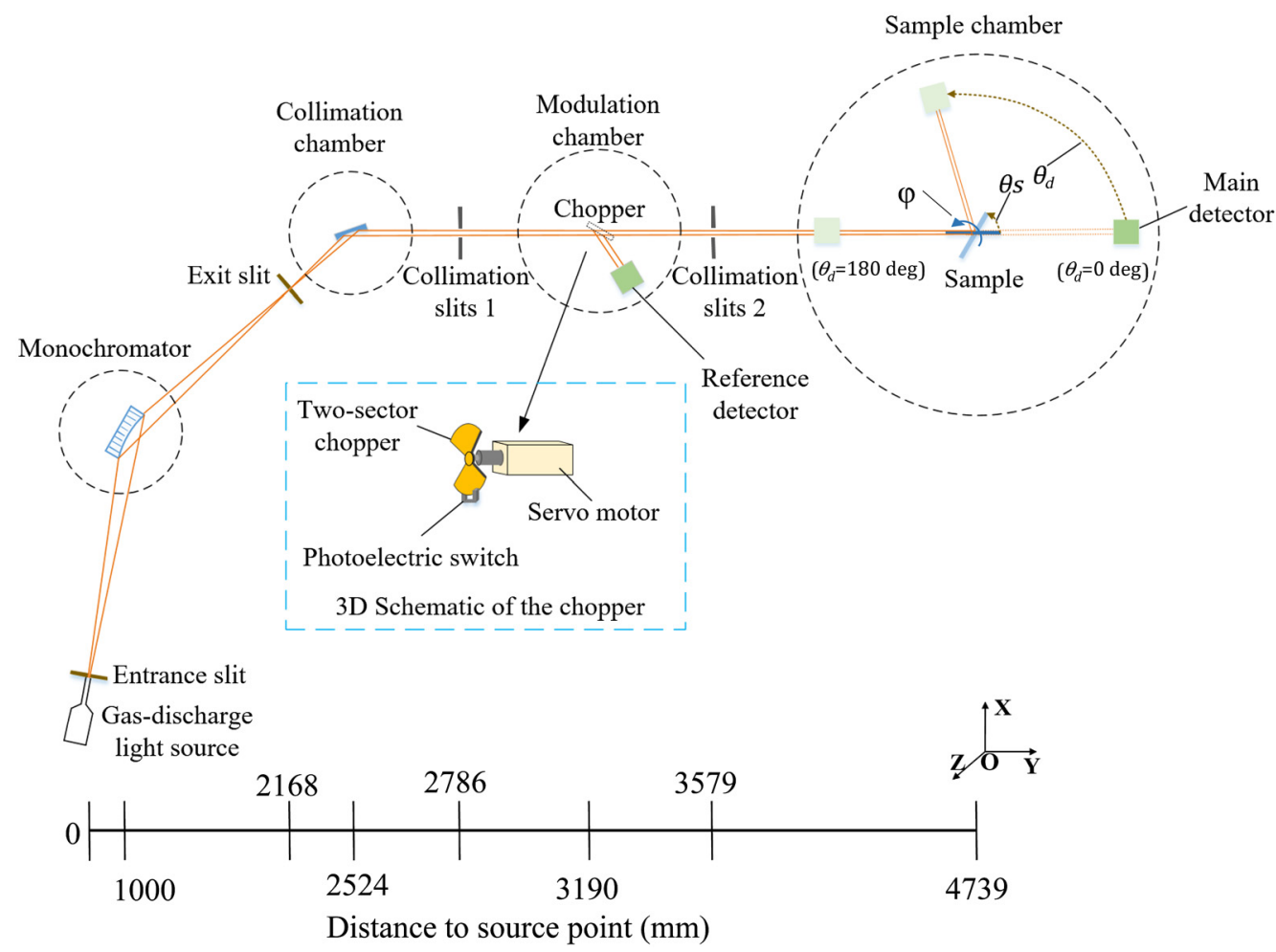

Fig. 1 Schematic of the reflectometer. The solid orange line represents the optical path. Inside the blue dotted box is a 3D schematic of the chopper disk and the servo motor.

\subsection{Specific Description of Each Unit}

\subsubsection{Gas-discharge light source}

In our system, we adopted an RF-induced gas-discharge lamp (FERMION INSTRUMENTS) based on local field plasma mechanism as the light source, which is electrode-free and ignitefree. A schematic of the lamp, as shown in Fig. 2, shows four parts in general: (1) a solid RF source with the central working frequency of $433 \mathrm{MHz}$ and the maximum power output of $200 \mathrm{~W}$. The optimal working frequency should couple with the position of focused electric field

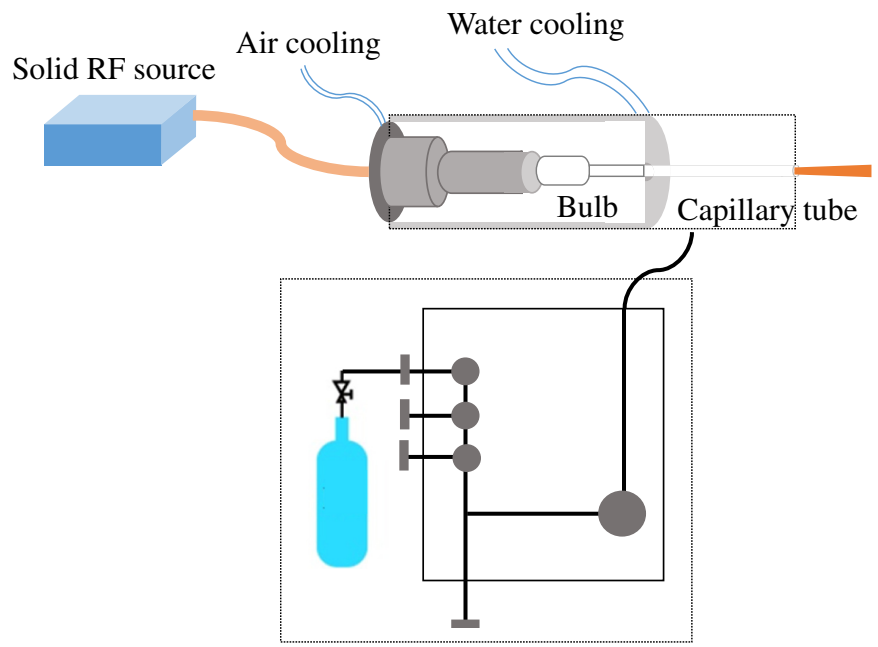

Gas supply system

Fig. 2 Schematic of the gas-discharge light source. 
Yu et al.: Laboratory-based reflectometer using line spectra of an RF-induced gas-discharge lamp...

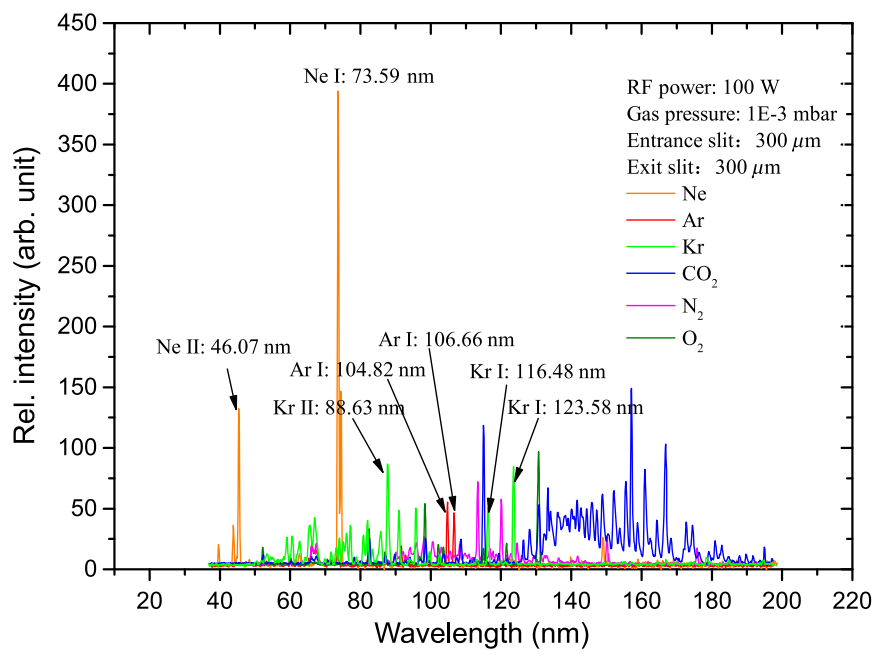

Fig. 3 Spectra of several atomic and melecular gases with $300-\mu \mathrm{m}$ entance and exit slit of the monochromator.

and should be fine adjusted according to the gas type and working pressure. (2) The capillary design is optimized to the resonant cavity which consists of a bulb and a tube. The bulb is used as the main gas discharge area, and the tube is used as an entrance for the gas inlet and access for the light transmission. (3) Gas supply system which ensures pure gases and controllable gas pressure. (4) Cooling system including water cooling and air cooling.

The lamp has a small weight (only $3 \mathrm{~kg}$ ) and it can accommodate helium, neon, argon, krypton, xenon, nitrogen, oxygen, etc., or a mix of the gases. By measuring the intensity of the exit EUV light beam from the tube, it was estimated that more than $5 \mathrm{E}+12$ photons could be generated per second for Ne II spectral line (46.07 $\mathrm{nm}$ and around) when the RF power of $100 \mathrm{~W}$ and neon gas pressure of $2 \mathrm{E}-4$ mbar was used. The divergence of the exit light beam is only about 2 deg which is very small and makes it compatible with monochromators. Using the spectral lines or relatively strong continuous spectra of the gases, the reflectometer is able to operate in the wavelength bands from 30 to $200 \mathrm{~nm}$. Such a broad working band is exciting because the probe beam in FUV band is relatively difficult to be acquired in laboratory-based reflectometers. ${ }^{36}$ The effect of higher diffraction order can be greatly reduced when only the discrete characteristic lines of the working gases are used for tests.

Figure 3 shows discharging spectra from 30 to $200 \mathrm{~nm}$ of several gases including neon, argon, krypton, carbon dioxide, nitrogen, and oxygen. Some strong characteristic lines are marked with the electronic energy level transitions and the corresponding wavelength available in the NIST website. All spectra were measured with the entrance and exit slits of $300 \mu \mathrm{m}$.

\subsubsection{Toroidal grating monochromator}

Horiba TGM1200 was adopted as the monochromator with a 1-m focal length and $146 \mathrm{deg}$ deviation angle between the entrance and exit arms. In the chamber, there are two switchable varied-line-spacing gratings working for different wavelength range, one is a 250 -gr/mm grating coated with Pt used for 50- to 200-nm wavelength range, while another Au coated grating with line density of $950 \mathrm{gr} / \mathrm{mm}$ is of high efficiency in 15 - to $50-\mathrm{nm}$ wavelength range. ${ }^{37}$ Both gratings are concave toroidal with the slope errors controlled at about 0.2 arc sec both in the directions parallel and perpendicular to the grooves, to ensure excellent performance for correcting aberrations and reducing the quantity of focusing mirrors in the optic path, thus the resolution and throughput of the monochromator can be improved.

In Fig. 4, we studied how the spectral resolution and exit light intensity vary with the slit width of the monochromator. With different configured entrance and exit slit widths, the complete intensity peaks of the characteristic lines are measured by a Si photodiode detector positioned right behind the exit slit. To maintain the spectral scanning with a step of $0.06 \mathrm{~nm}$, the 


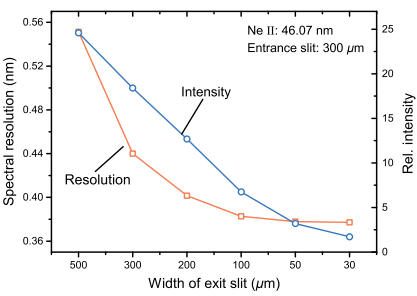

(a)

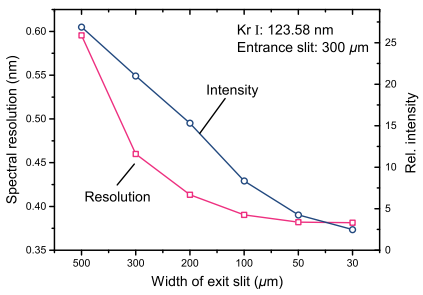

(d)

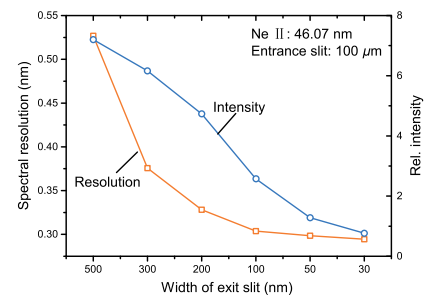

(b)

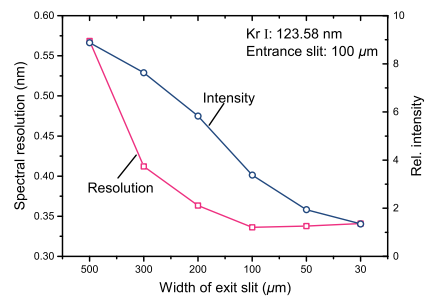

(e)

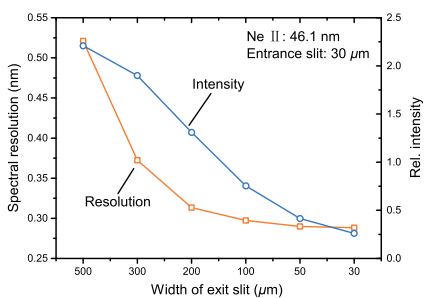

(c)

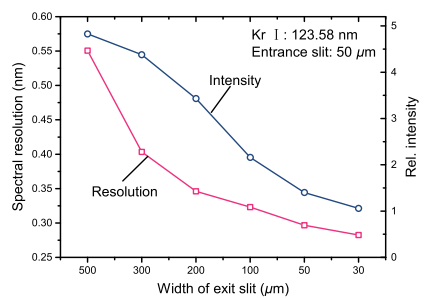

(f)

Fig. 4 Spectral resolution and exit beam intensity variation as a function of exit slit width at $46.07 \mathrm{~nm}$ with entrance slit width of (a) 300, (b) 100 , (c) 30 , and at $123.58 \mathrm{~nm}$ with entrance slit width of (d) 300, (e) 100, and (f) $50 \mu \mathrm{m}$.

grating rotation and data collection are controlled by a customized LabVIEW program. For each characteristic line, the RF power and gas pressure are required to keep stable during measurement. The spectral resolution as presented by FWHM is deduced by fitting the peaks with a Gauss profile, and the monochromatic light intensities are determined by the peak values. The spectral resolution reaches as high as $0.27 \mathrm{~nm}$ at $46.07 \mathrm{~nm}$ with $10-\mu \mathrm{m}$ entrance slit and $30-\mu \mathrm{m}$ exit slit, and $0.28 \mathrm{~nm}$ at $123.58 \mathrm{~nm}$ with $50-\mu \mathrm{m}$ entrance slit and $30-\mu \mathrm{m}$ exit slit, but the intensity of the exit beam was too weak to be used for reflectivity measurements presently using the photodiode detector. Considering the fact that we used characteristic lines rather than continuous spectra, the width of the slits does not affect the spectral resolution so much. So we adjusted the entrance and exit slit at $300 \mu \mathrm{m}$ where the spectral resolution moderately decreases to $0.44 \mathrm{~nm}$ at $46.07 \mathrm{~nm}$ and $0.46 \mathrm{~nm}$ at $123.58 \mathrm{~nm}$ with the intensity increased by more than 100 times and 20 times, respectively, compared with the situation of the highest resolution. With the entrance and exit slit of $300 \mu \mathrm{m}$, the throughput of the monochromator is about $4.6 \times 10^{10}$ photons per second (PPS) at $46.07 \mathrm{~nm}$ and $8.2 \times 10^{9}$ PPS at $123.58 \mathrm{~nm}$.

\subsubsection{Collimation chamber}

A collimation chamber is coupled to the monochromator exit flange. Inside this chamber, an $\mathrm{Au}$ coated customizing designed toroidal mirror, with $11.3 \mathrm{arc} \mathrm{sec}$ slope error in the long side direction and $53.1 \mathrm{arc}$ sec in the short side direction, was mounted on a six-dimensional (6D) adjustment platform. The beam exit from the monochromator is directed onto the mirror with incident angle varied from 69.34 deg to $70.66 \mathrm{deg}$. Within this incident angle range, the Au film keeps relatively high reflectivity in 30- to 200-nm wavelength region, as shown in Fig. 5. The 6D adjustment platform enables the mirror to be precisely transferred along $X$ and $Y$ axis ( $X$ axis is perpendicular to mirror surface and $Y$ axis is parallel to mirror surface), transferred vertically along $Z$ axis, rotated with respect to $Z$ axis, tipped with respect to the $X$ axis and tilted with respect to the $Y$ axis. The stability of the platform has been proved by observing the position of the beam using a UV CCD mounted on the sample chamber far away and no obvious shift has been found for several months.

The incident beam is reflected by the collimator mirror with an approximate area of $8 \mathrm{~mm} \times$ $8 \mathrm{~mm}$ perpendicular to the optical path, which is further collimated by two sets of collimation slits sited at the positions of about 230 and $1260 \mathrm{~mm}$ away from the mirror. The collimator mirror and two slits reduced the divergent angle of beam from $1.32 \mathrm{deg}$ to $-0.01 \mathrm{deg}$ in meridian direction (in the $X O Y$ plane) and from $1.23 \mathrm{deg}$ to $0.08 \mathrm{deg}$ in sagittal direction (in the $\mathrm{XOZ}$ plane), 
Yu et al.: Laboratory-based reflectometer using line spectra of an RF-induced gas-discharge lamp...

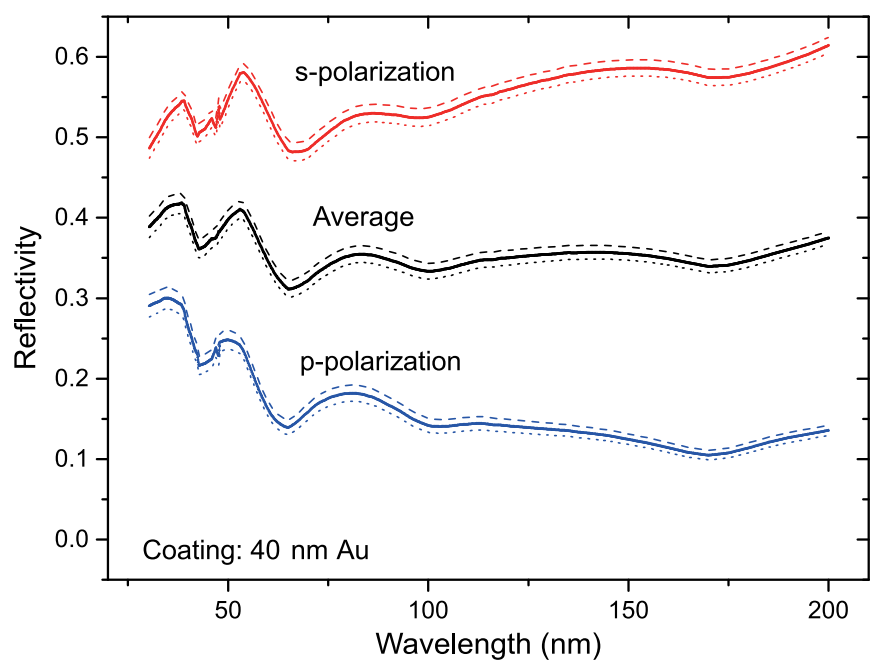

Fig. 5 S-polarized (red lines), p-polarized (blue lines) and average (black lines) reflectivity of $\mathrm{Au}$ coating in 30- to 200-nm wavelength range at minimum incident angle of 69.34 deg (dotted lines), central incident angle of $70 \mathrm{deg}$ (solid lines) and maximum incident angle of $70.66 \mathrm{deg}$ (dash lines).

according to experimental measurements. As the angular scans of the samples are performed in $X O Y$ plane, the divergence in the sagittal direction does not affect the angular resolution in the experiments.

\subsubsection{Modulation/monitor chamber}

To measure extremely weak reflected beam with the help of lock-in amplifiers, it is necessary to modulate the temporal quasi-continuous light into periodic. For creating periodically modulated light as well as monitoring the light intensity, a two-sector optical chopper disk was mounted in the chamber with Au-coated polished glass cemented on the blades. On the one hand, the periodic signal detected by a groove photoelectric sensor mounted under the blades serves as a reference input for lock-in amplifiers, and the lock-in amplifiers then single out the component of the signal at the reference frequency using a phase-sensitive detection technique so that very small AC signals down to a few pico-amperes can be measured. In our system, the maximum modulating frequency is $60 \mathrm{~Hz}$ with the disk rotating 30 revolutions per second. On the other hand, the chopper functions as a beam splitter. The disk chops the light beam at an incident angle of $70 \mathrm{deg}$, and the reflected reference beam was detected by a Si photodiode (AXUV100G type) to monitor the intensity of the incident beam.

\subsubsection{Sample chamber}

The sample chamber is a 1.5-m high and 1.2-m diameter chamber housing a high-precision translation stage consisting of a three-dimensional linear stage, an angular stage, and a goniometer assembly with six degrees of freedom which were marked in Fig. 1, including X (vertical to the optical path direction in horizontal surface), $Y$ (along the optical path direction), $Z$ (vertical to horizontal surface), $\varphi$ (sample inclination), $\theta_{s}$ (sample rotation) and $\theta_{d}$ (detector rotation), respectively. The $\theta_{s}$ and $\theta_{d}$ have concentric rotation axis perpendicular to the XOY plane with resolution within $4^{\prime \prime}$ from $0 \mathrm{deg}$ to $360 \mathrm{deg}$ range. The sample is cemented on a bracket that should be inserted into a fixed slot structure. The sample surfaces were initially parallel to the YOZ plane which corresponds to $\theta_{s}=0 \mathrm{deg}$. Another twin Si photodiode (AXUV100G type) is used as the detector. The detector was mounted on a rotation arm assembly which was about $430 \mathrm{~mm}$ away from the rotation axis and was initially placed at the end of the light beam right behind the sample, which was set as $\theta_{d}=0 \mathrm{deg}$, as shown in Fig. 1. The detector can rotate dependently or independently with the sample. $X, Y$, and $Z$ motions have a resolution of $3 \mu \mathrm{m}$ over its range of $\pm 50 \mathrm{~mm}$. The tilt motion controls the sample inclination relative to $\mathrm{Y}$ axis, and 
its resolution is better than $3^{\prime \prime}$ within the angular range of $\pm 20 \mathrm{deg}$. Currently, the samples are cemented on a bracket alone $Z$ direction, thus the $Z$ motion can be used to switch the samples. $X$ and $Y$ motions would be applied if the samples need to be translated out of the beam. The stage allows a maximum sample diameter of $100 \mathrm{~mm}$ and a weight capacity of $30 \mathrm{~kg}$.

The sample chamber connects with the former chambers by a manual valve. When closing the manual valve, the sample chamber can be vented alone, which is quite convenient for fast changing samples.

\section{Reflectometer Adjustment}

To achieve optimal performance of the reflectometer, precise alignment of the system is necessary. To begin with, a benchmark of the optical axis for subsequent adjustment should be determined.

As shown in Fig. 6(a), the two positions of the detector at $0 \mathrm{deg}$ and $180 \mathrm{deg}$, respectively, were considered as the datum points to determine the optical axis of the reflectometer, which was then recorded using a theodolite. Afterward, a rough alignment of the system including the chambers and collimation slits was carried out with the help of the theodolite and a compact laser mounted at the end of sample chamber and directed reversely along the optical axis toward the theodolite, as shown in Fig. 6(b). Chambers are connected by bellows which made it possible to shift their positions slightly. Precise alignment was achieved with an auto-collimation telescope, which was located behind the end of sample chamber. Slightly and repetitively adjustments of collimation slits, collimator mirror, slits of monochromator, and the capillary tube of EUV light source were performed progressively, until they all positioned right in the center of the telescope field-of-view, as shown in Fig. 6(c).

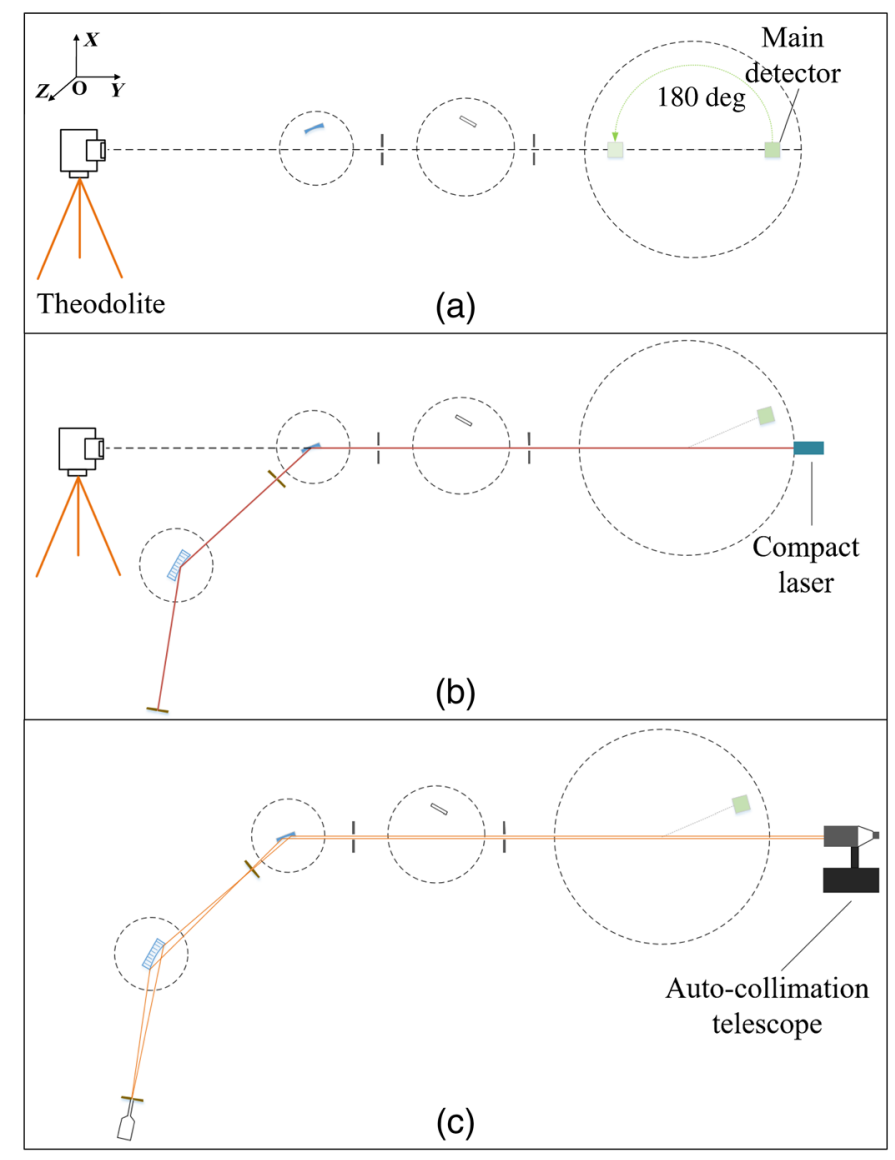

Fig. 6 Adjustment process: (a) and (b) represent rough alignment with a theodolite and a compact laser, and (c) represents precise alignment with an auto-collimation telescope. 


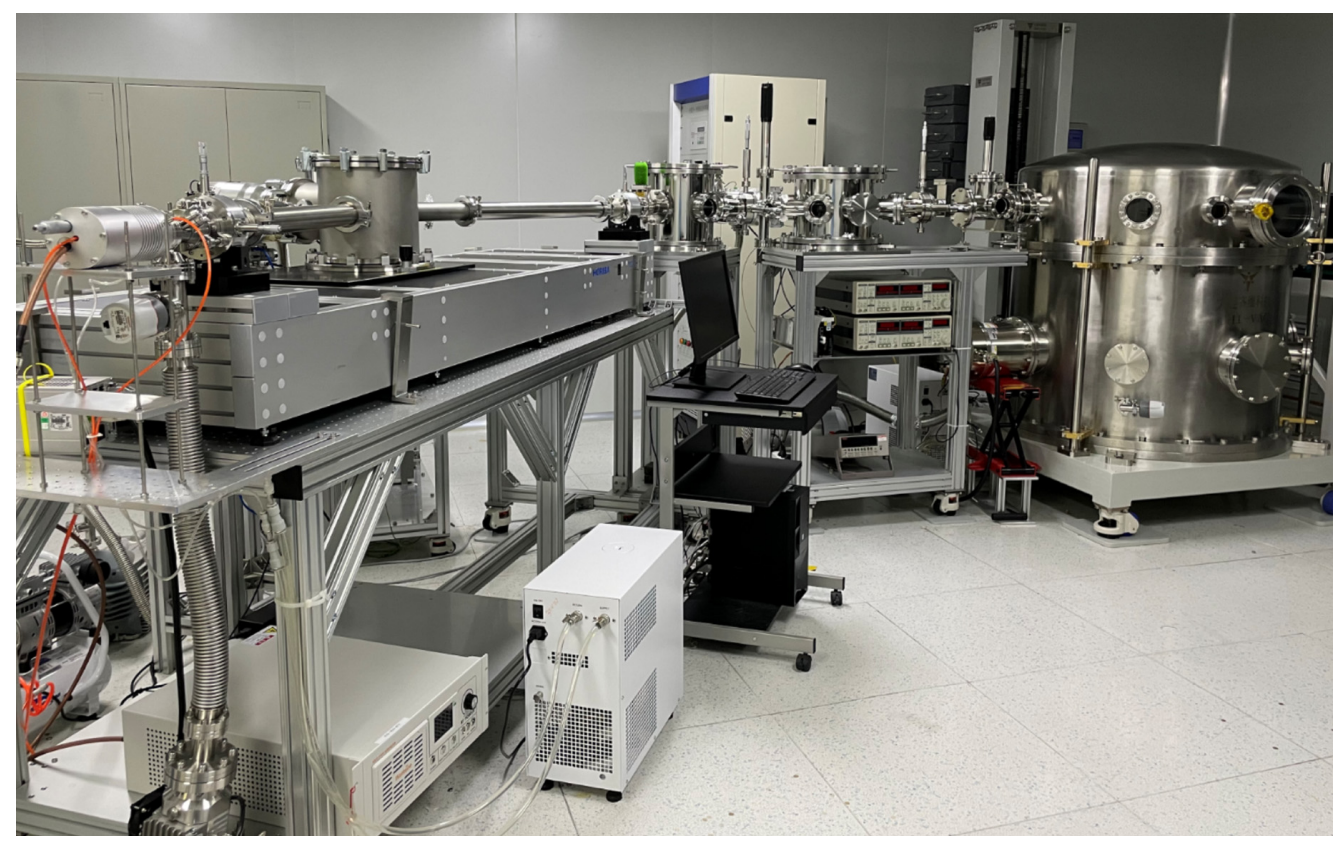

Fig. 7 Photograph of the system.

The 6D platform of collimator mirror allowed for a final adjustment of the beam direction. The criterion of adjusting the $6 \mathrm{D}$ platform is that the reflected beam is nearly parallel in the meridional plane. By precisely adjusting each unit of the system, the detecting beam light intensity can reach an optimal value, and a bright image of the monochromator slit with clear outline and minimal aberration can be acquired. A photograph of the system after adjustment is shown in Fig. 7. All the components of the system are mounted on the frames with supporting structures, each consisting of a wheel and a block that can be switched and locked by a gear-shape valve. During alignment, the wheel is used for flexible moving, and for long-term running, the block is lowered down to maintain stability. The reliability has been corroborated by observing through the above-mentioned UV CCD mounted on the sample chamber.

The measured and simulated light intensity distribution and cross-section size of the beam at the detector position are shown in Figs. 8(a) and 8(b), respectively. The asymmetry of the intensity distribution can be found both in the experimental and simulation results due to the aberrations caused by the gratings and collimating mirror. As for the spot size, the experimental measured spot is about $1.6 \mathrm{~mm} \times 4.7 \mathrm{~mm}$, which is larger than simulated spot of

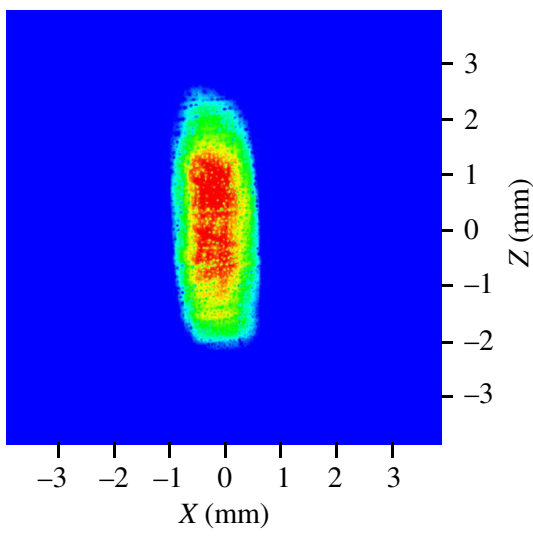

(a)

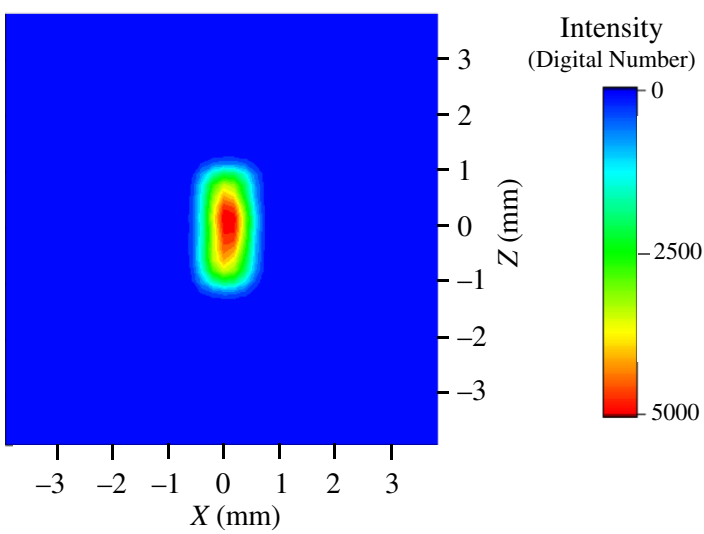

(b)

Fig. 8 2D-map of the beam profile at the detector position in case of (a) experimental acquired and (b) simulation. 
$1.3 \mathrm{~mm} \times 2.5 \mathrm{~mm}$. The quality of the beam is influenced by the slope/figure errors of the gratings and the collimator mirror. In addition, according to the simulation by ZEMAX and the experimental observations, the beam quality is also sensitively affected by the adjustment inaccuracy, such as the position and orientation of the collimator mirror. For this reflectometer, a collimated beam is highly concerned of. So the negative effects of the slope/figure errors and the adjustment inaccuracy can be effectively reduced using two sets of collimation slits to improve the beam quality.

\section{Experimental Results and System Performance}

The procedure for the reflectivity measurement includes two steps, the sample orientation alignment and the reflectivity measurements. For testing the performance of this system, two samples of $\mathrm{Sc} / \mathrm{Si}$ multilayer and $\mathrm{Al} / \mathrm{LiF} / \mathrm{MgF}_{2}$ film were chosen. The measurement procedure and reflectivity results will be given as follows.

\subsection{Sample Orientation Alignment}

Adjustment of sample orientation is a necessary step before reflectivity measurements to make sure the sample surface coincides with the rotation axis and the reflected light beam incident on the center of the detector.

There are three steps in general for the sample orientation alignment. First of all, move the sample out of the beam path and scan the detector from $-1 \mathrm{deg}$ to $1 \mathrm{deg}$ with step of $0.1 \mathrm{deg}$. As shown in Fig. 9(a), the red star at the midpoint of the platform corresponds to $\theta_{d}=0$ deg with respect of probe beam direction.

Secondly, move in the sample along the $X$ direction perpendicular to the incident beam. Repeatedly move the sample along the $X$ direction with a step of $0.1 \mathrm{~mm}$ and rotate around the rotation axis with step of $0.1 \mathrm{deg}$, as shown in Figs. 9(b) and 9(c), respectively, until the sample cut off half intensity of the incident probe beam. The red star in Fig. 9(b) at the half of the slope corresponds to the exact X value. The red star in Fig. 9(c) shows the maximum intensity where the $\theta_{s}$ was set as $0 \mathrm{deg}$. The asymmetry of the light intensity slope in Fig. 9(c) is probably due to the non-uniform intensity distribution of the incident beam, which

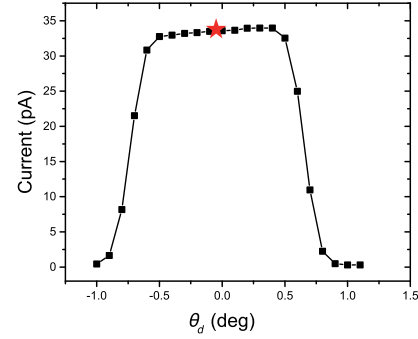

(a)

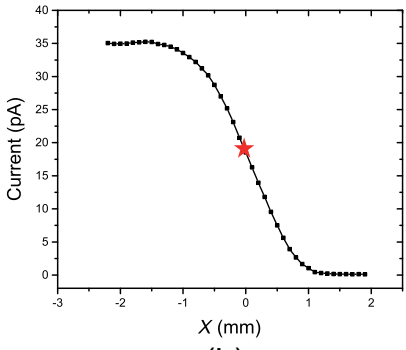

(b)

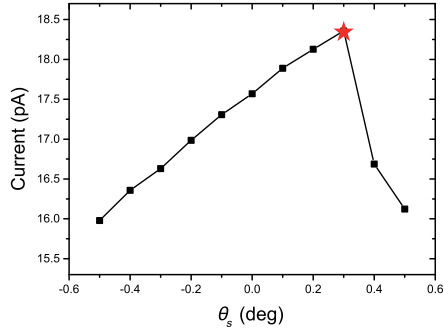

(c)

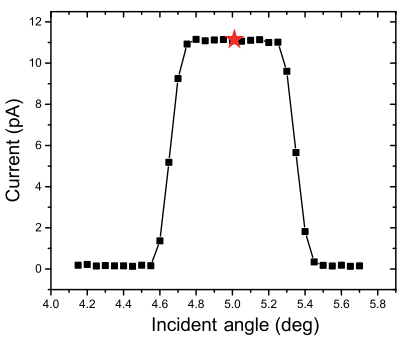

(d)

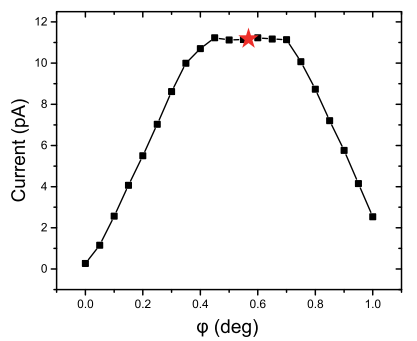

(e)

Fig. 9 Sample attitude adjustment. (a) $\theta_{d}$ scan, (b) $X$ scan, (c) $\theta_{s}$ scan at initial position, (d) $\theta_{s}$ scan at normal incident $5 \mathrm{deg}$, and (e) $\varphi$ scan. 
Yu et al.: Laboratory-based reflectometer using line spectra of an RF-induced gas-discharge lamp...

would cause the sample surface are not in strictly coincidence with the rotation axis and thus the position of the light spot incident on the detector would shift with large rotation angle.

To solve the uncertainty, we need to slightly compensate the offset and calibrate again including the sample rotation angle and inclination at its working angle, to make sure the reflected light beam incident on the center of the photodiode both in the vertical and horizontal direction. Figures 9(d) and 9(e) gives $\theta_{s}$ scan around the working incident angle and $\varphi$ scan for calibrating inclination, respectively. The red stars at the midpoint of the platform in Figs. 9(d) and 9(e) represent the calibrated nominal $5 \mathrm{deg}$ and the exact $\varphi$ value, respectively.

\subsection{Sample Reflectivity Measurements}

\subsubsection{Angular dependence of reflectivity}

$\mathrm{Sc} / \mathrm{Si}$ multilayer has excellent reflectivity at the wavelength of 35 to $50 \mathrm{~nm}$ and is expected to be applied in the solar EUV telescope for reflecting Ne VII line $(46.5 \mathrm{~nm})$ at normal incidence angle. $^{38}$ The tested sample was designed as $[\mathrm{Si}(6.77 \mathrm{~nm}) / \mathrm{Sc}(15.65 \mathrm{~nm})]_{20} / \mathrm{Si}$-sub with a top silicon oxide layer estimated to be around $2 \mathrm{~nm},{ }^{39}$ to work at $46.5 \mathrm{~nm}$ with the normal incidence angle of $4.6 \mathrm{deg}$. The sample was deposited on a $20 \mathrm{~mm} \times 20 \mathrm{~mm}$ polished Si wafer by DC magnetron sputtering and stored with $15 \%$ humidity.

Its angular dependence of the reflectivity was measured and shown in Fig. 10 from 3 deg to $20 \mathrm{deg}$ incident angle with a step of $1 \mathrm{deg}$. At each angle, both the reflected light intensity and reference light intensity was sampling with $512 \mathrm{~Hz}$ and subtracted by background signal. The reflected light intensity was normalized to the reference signal. Theoretical reflectivity calculated by IMD software ${ }^{40}$ was also given in Fig. 10 which has preliminarily verified the experimental results were reasonably close to the expected values.

\subsubsection{Spectral dependence of reflectivity}

$\mathrm{Al} / \mathrm{LiF} / \mathrm{MgF}_{2}$ film was distinguished by its excellent reflective performance in FUV band thus worth researching for further applications in many astrophysical missions. We have tested a piece of $\mathrm{Al} / \mathrm{LiF} / \mathrm{MgF}_{2}$ film sample utilizing relatively strong characteristic line spectrum of several atomic and molecular gases. For calibration, we also measured its reflectivity at BESSY-II synchrotron and HLS, which is given in Fig. 11. Compared with average test value of BESSY-II synchrotron, the deviation is $<9 \%$, while compared with tests of HLS, the deviation is $<5 \%$. The individually shift deviations may be caused by some very weak spectral lines,

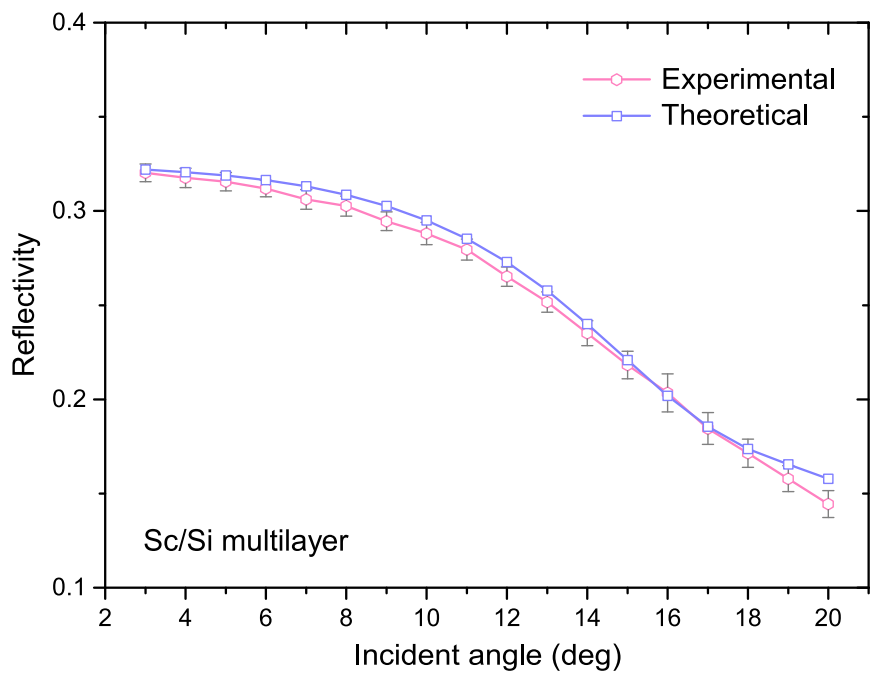

Fig. 10 Reflectivity curve with changing incident angle from 3 deg to 20 deg. 
Yu et al.: Laboratory-based reflectometer using line spectra of an RF-induced gas-discharge lamp...

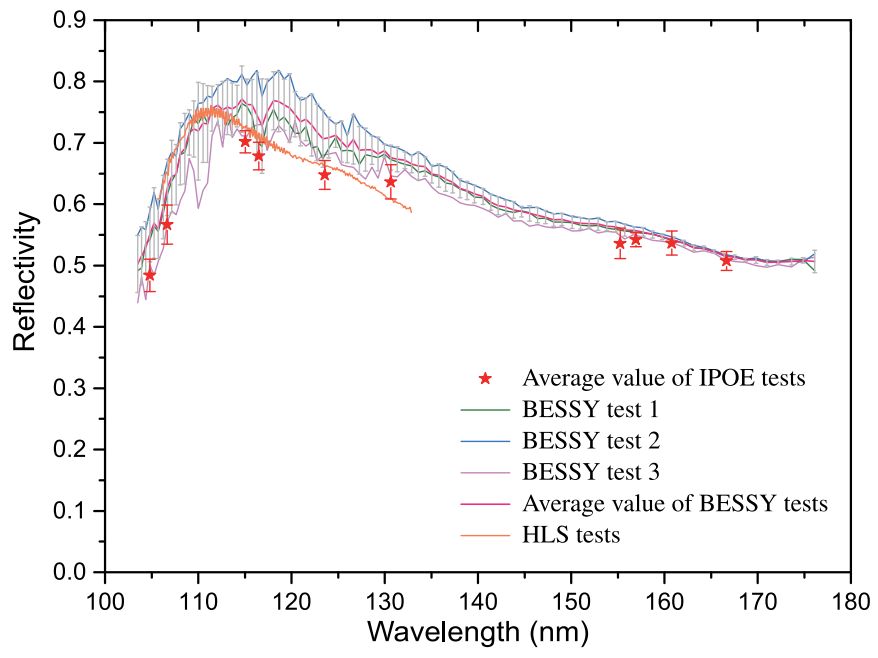

Fig. 11 Reflectivity value of $\mathrm{Al} / \mathrm{LiF} / \mathrm{MgF}_{2}$ film at different wavelength and compared with tests from BESSY-II and HLS.

consequently, the reflected light intensity would be affected slightly by the fluctuation of the background intensity. At wavelength around $105 \mathrm{~nm}$, results of HLS are relatively unreliable because of the influence of higher diffraction orders.

\subsubsection{Stability and repeatability}

For verifying system stability, we have performed the same experiment for nine times and acquired a $0.5 \%$ RMS value. In addition, with all the experimental conditions unchanged, we carried out the same experiments for days' interval to test system repeatability. Results have demonstrated that the repeatability is better than $2 \%$.

\section{Summary}

The configuration, adjustment, and test results of a reflectometer used for studying optical properties of films in EUV and FUV wavelength band at IPOE laboratory are described. The system mainly consists of an RF-produced gas-discharge light source, a toroidal grazing monochromator, a collimator chamber, a modulation chamber and a sample chamber. After precise and repetitive adjustments of the system, experiments including sample orientation alignments and reflectivity measurements were carried out using the reflectometer.

For testing the performance of this system, we gave an angular scan reflectivity measurement of $\mathrm{Sc} / \mathrm{Si}$ multilayer and compared the results with theoretical values. We also measured the spectral dependence of reflectivity of $\mathrm{Al} / \mathrm{LiF} / \mathrm{MgF}_{2}$ film, then compared the results with test data from BESSY-II and HSL, respectively. The measurement deviation between our system and BESSY-II is $<9 \%$ while HLS is $<5 \%$. Results have proven the stability and reliability of the system with repeatability of $2 \%$ and accuracy better than $0.5 \%$. Construction of the reflectometer is expected to obviously shorten the turn-around time of the research and development process of optical coatings at IPOE.

Currently, some spectral lines with much weaker intensity are unable to be applied for reflectivity measurements, and the probe light beam hasn't been linearly polarized yet. In the near future, we plan to substitute the photodiode with channel electron multiplier, so that much weaker characteristic lines and continuous spectra can be used to measure the reflectivity of optical coatings. A four-mirror polarizer will be mounted in the system to produce s-polarized light. At the same time, the probe beam spot on sample surface can be greatly reduced in size which will allow reliable measurements of the samples with relatively large surface peak-valley value. 
Yu et al.: Laboratory-based reflectometer using line spectra of an RF-induced gas-discharge lamp...

\section{Acknowledgments}

This work was supported in part by the National Natural Science Foundation of China under Grant Nos. 11875203 and 12003016, and in part by the Strategic Priority Research Program of the Chinese Academy of Sciences under Grant No. XDA15018400.

\section{References}

1. A. Bayer et al., "Material analysis with EUV/XUV radiation using a broadband laser plasma source and optics system," Proc. SPIE 7360, 736004 (2009).

2. P. Zuppella et al., "Characterization of optical materials for EUV spectral region: methods and analysis," AIP Conf. Proc. 2146, 020024 (2019).

3. L. Mezi et al., "ENEA EUV discharge produced plasma source: diagnostics, characterization and applications," in ECPD, Vol. 125 (2015).

4. S. Bollanti et al., "Space-and time-resolved diagnostics of the ENEA EUV dischargeproduced-plasma source used for metrology and other applications," High Power Laser Sci. Eng. 3, e29 (2015).

5. P. Wachulak et al., "Advances in microscopic imaging at the nanoscale using soft X-rays and extreme ultraviolet (EUV) from a compact laser plasma source," Proc. SPIE 11076, 1107606 (2019).

6. M. Bitter et al., "Objective for EUV microscopy, EUV lithography, and x-ray imaging," U.S. Patent No. 9,329,487. 3 (2016).

7. J. V. Schoot et al., "The future of EUV lithography: continuing Moore's law into the next decade," Proc. SPIE 10583, 105830R (2018).

8. K. Kasahara et al., "Recent progress in nanoparticle photoresists development for EUV lithography," Proc. SPIE 9776, 977604 (2016).

9. H. W. Moos et al., "FUSE: lessons learned for future FUV missions," Proc. SPIE 5488, $1-12$ (2004).

10. V. Domingo, "SOHO, Yohkoh, Ulysses and trace: the four solar missions in perspective, and available resources," Astrophys. Space Sci. 282, 171-188 (2002).

11. G. Rottman et al., "The Solar Radiation and Climate Experiment (SORCE): mission description and early results," Solar Phys. 230, 1-2 (2005).

12. J. R. Lemen et al., "The atmospheric imaging assembly (AIA) on the solar dynamics observatory (SDO)," Solar Phys. 275, 17-40 (2012).

13. J. -P. Delaboudinière et al. "EIT: extreme-ultraviolet Imaging Telescope for the SOHO mission," Solar Phys. 162, 291-312 (1995).

14. M. Wen et al., "High efficiency carbon-based multilayers for LAMP at $250 \mathrm{eV}$," Proc. SPIE 9603, 96031A (2015).

15. "Thermo Scientific GENESYS Vis and UV-Vis spectrophotometers," Thermo Fisher Scientific Inc., https://www.thermofisher.com (2018).

16. "LAMBDA series spectrophotometers," PerkinElmer, Inc., https://www.perkinelmer.com .cn/category/molecular-spectroscopy-instruments (2013).

17. "Vacuum Ultraviolet Monochromators and Spectrometers Data Sheet," McPherson, https:// mcphersoninc.com/spectrometers/vuvuvvis/vuvuvvis.html (2017).

18. "H20 UVL Monograph for Far Ultraviolet," HORIBA, https://pdf.directindustry.com/pdf/ horiba-scientific/h20-uvl-monochromator-far-ultraviolet/25366-458611.html.

19. R. Cubitt and G. Fragneto, "D17: the new reflectometer at the ILL," Appl. Phys. A 74, S329S331 (2002).

20. E. M. Gullikson et al., "Recent developments in EUV reflectometry at the advanced light source," Proc. SPIE 4343, 363-373 (2001).

21. A. Sokolov et al., "An XUV optics beamline at BESSY II," Proc. SPIE 9206, 92060J (2014).

22. S. Nannarone et al., "The BEAR beamline at elettra," AIP Conf. Proc. 705, 450-453 (2004).

23. H. Iguchi et al., "Development of a reflectometer for a large EUV mirror in NewSUBARU," Proc. SPIE 9658, 965819 (2015).

24. R. A. Bosch et al., "SURF III—a new electron storage ring at NIST," AIP Conf. Proc. 521, 383 (2000). 
Yu et al.: Laboratory-based reflectometer using line spectra of an RF-induced gas-discharge lamp...

25. U Flechsig et al., "The optics beamline at the Swiss Light Source," Nucl. Instrum. Methods Phys. Res., Sect. A 609, 281-285 (2009).

26. K. Sawhney et al., "A test beamline on diamond light source," AIP Conf. Proc. 1234, 387-390 (2010).

27. A. Sokolov et al., "At-wavelength metrology facility for soft X-ray reflection optics," Rev. Sci. Instrum. 87, 052005 (2016).

28. A. Sokolov et al., "Efficient high-order suppression system for a metrology beamline," J. Synchrotron Rad. 25, 100-107 (2018).

29. S. A. Garakhin et al., "High-resolution laboratory reflectometer for the study of x-ray optical elements in the soft and extreme ultraviolet wavelength ranges," Rev. Sci. Instrum. 91, 063103 (2020).

30. L. R. Marcos et al., "Advances in far-ultraviolet reflective and transmissive coatings for space applications," Proc. SPIE 9912, 99122E (2016).

31. U. Hinze et al., "High-accuracy EUV reflectometer," Proc. SPIE 6517, 651732 (2007).

32. S. Nakayama et al., "Soft X-ray reflectometer with a laser-produced plasma source," Phys. Scr. 41, 754 (1990).

33. E. M. Gullikson et al., "A soft x-ray/EUV reflectometer based on a laser produced plasma source," J. X-Ray Sci. Technol. 3, 283-299 (1992).

34. D. L. Windt et al., "Soft x-ray reflectometry of multilayer coatings using a laser-plasma source," Proc. SPIE 1547, 144-158 (1992).

35. J. A. Aznárez, J. I. Larruquert, and J. A. Méndez "Far-ultraviolet absolute reflectometer for optical constant determination of ultrahigh vacuum prepared thin films," Rev. Sci. Instrum. 67, 497 (1996).

36. "Operation Manual for Multi-Gas Ultra-High Flux VUV Source BL1010s," Fermion Instruments (Shanghai) Co., Ltd., http://en.fermi.com/productclass_3.

37. "TGM1200-HV-2R operating instruction," HORIBA, 2020, 2nd version, https://pdf .directindustry.com/pdf/horiba-scientific/tgm-series-toroidal-grating-monochromators/ 25366-613125.html.

38. J. Wu et al., "Structure and stability of $\mathrm{Sc} / \mathrm{Si}$ multilayers with different thickness ratio for wavelength at 46.5 nm," Proc. SPIE 11491, 1149106 (2020).

39. J. Gautier et al., "Characterization and optimization of magnetron sputtered Sc/Si multilayers for extreme ultraviolet optics," Appl. Phys. A 88, 719-725 (2007).

40. D. L. Windt, "IMD—software for modeling the optical properties of multilayer films," Comput. Phys. 12, 360 (1998).

Yue Yu is currently a $\mathrm{PhD}$ candidate from Tongji University. She received her BS degree from Ocean University of China in 2017. Her current research interests include EUV/x-ray reflectometers.

Wenbin Li is a professor at the School of Physics Science and Engineering of Tongji University. He received his BS degree in physics from Anhui Normal University in 1995 and his $\mathrm{PhD}$ in physics from the University of Science and Technology of China in 2004. His current research interests include x-ray multilayer mirrors, soft x-ray polarimetry, EUV/x-ray reflectometer, and EUV/x-ray damage.

Zhanshan Wang is a SPIE fellow and a professor at Tongji University. He received his MS degree in optics from Changchun Institute of Optics and Fine Mechanics, CAS, in 1988, and his $\mathrm{PhD}$ in optics from Shanghai Institute of Optics and Fine Mechanics, CAS, in 1996. He is the author of more than 150 journal papers. His current research interests include soft x-ray sources, optics in EUV and $\mathrm{x}$-ray region, optical coatings for lasers and optical systems.

Biographies of the other authors are not available. 